Ferrata Storti Foundation

\section{Measurable residual disease monitoring provides insufficient lead-time to prevent morphological relapse in the majority of patients with core-binding factor acute myeloid leukemia}

\author{
Robert Puckrin, ${ }^{1,2}$ Eshetu G. Atenafu, ${ }^{3}$ Jaime 0. Claudio, ${ }^{1}$ Steven Chan, ${ }^{1,2}$ Vikas \\ Gupta,,$^{1,2}$ Dawn Maze, ${ }^{1,2}$ Caroline McNamara, ${ }^{1,2}$ Tracy Murphy, ${ }^{1,2}$ Andre C. \\ Schuh,,$^{1,2}$ Karen Yee, ${ }^{1,2}$ Hassan Sibai, ${ }^{1,2}$ Mark D. Minden, ${ }^{1,2}$ Cuihong Wei, ${ }^{1,4}$ Tracy \\ Stockley, ${ }^{1,4}$ Suzanne Kamel-Reid ${ }^{1,4}$ and Aaron D. Schimmer ${ }^{1,2}$ \\ ${ }^{1}$ Princess Margaret Cancer Centre, University Health Network; ${ }^{2}$ Department of Medicine, \\ University of Toronto; ${ }^{3}$ Department of Biostatistics, Princess Margaret Cancer Centre, \\ University Health Network and ${ }^{4}$ Department of Clinical Laboratory Genetics, Laboratory \\ Medicine Program, University Health Network, Toronto, Canada
}

Volume 106(1):56-63

\section{ABSTRACT}

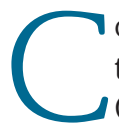
ore-binding factor acute myeloid leukemia is characterized by $t(8 ; 21)$ or inv(16) and the fusion proteins RUNX1-RUNX1T1 and CBFB-MYH11. International guidelines recommend monitoring for measurable residual disease every 3 months for 2 years after treatment. However, it is not known whether serial molecular monitoring can predict and prevent morphological relapse. We conducted a retrospective singlecenter study of 114 patients in complete remission who underwent molecular monitoring with real-time quantitative polymerase chain reaction analysis of RUNX1-RUNX1T1 or CBFB-MYH11 transcripts every 3 months. Morphological relapse was defined as re-emergence of $>5 \%$ blasts and molecular relapse as $\geq 1$ log increase in transcript level between two samples. Over a median follow-up time of 3.7 years (range, 0.2-14.3), remission persisted in $71(62.3 \%)$ patients but $43(37.7 \%)$ developed molecular or morphological relapse. Patients who achieved $<3$ log reduction in RUNX1RUNX1T1 or CBFB-MYH11 transcripts at the end of chemotherapy had a significantly higher risk of relapse compared to patients who achieved $\geq 3$ $\log$ reduction $(61.1 \%$ vs. $33.7 \%, P=0.004)$. The majority of relapses $(74.4 \%$, $\mathrm{n}=32$ ) were not predicted by molecular monitoring and occurred rapidly with $<100$ days from molecular to morphological relapse. Molecular monitoring enabled the detection of impending relapse and permitted pre-emptive intervention prior to morphological relapse in only 11 (25.6\%) patients. The current practice of molecular monitoring every 3 months provided insufficient lead-time to identify molecular relapses and prevent morphological relapse in the majority of patients with core-binding factor acute myeloid leukemia treated at our institution. Further research is necessary to determine the optimal monitoring strategies for these patients.

\section{Introduction}

Core-binding factor (CBF) acute myeloid leukemia (AML) is recognized by the World Health Organization as a subtype of AML with recurrent genetic abnormalities characterized by the chromosomal abnormalities $\mathrm{t}(8 ; 21)$ or inv(16)/t(16;16). ${ }^{1}$ At the molecular level, these chromosomal alterations result in production of the fusion proteins RUNX1-RUNX1T1 in cases with $\mathrm{t}(8 ; 21)$ and CBFB-MYH11 in those with inv(16)/t(16;16). CBF-AML accounts for $10-15 \%$ of adult AML and tends to be associated with younger patient age and higher sensitivity to induction and consolidation chemotherapy. ${ }^{2,3}$ Despite a relatively favorable prognosis, up to $40 \%$ of patients with CBF-AML relapse. , $^{2,5}$ 
The presence of minimal or measurable residual disease $(\mathrm{MRD})$, defined as the presence of leukemic cells after treatment at levels of $1: 10^{4}$ to $1: 10^{6}$, predicts poor outcomes and impending relapse in AML. ${ }^{6-8}$ Molecular monitoring for $\mathrm{MRD}$ is well-established in hematologic malignancies with clearly defined disease markers, such as BCR-ABL1 in chronic myeloid leukemia and NPM1 in NPM1-mutated AML. With the ability of real-time quantitative polymerase chain reaction (RT-qPCR) to quantify transcript levels of RUNX1-RUNX1T1 and CBFB-MYH11, CBF-AML represents one of the other most well-established targets for MRD monitoring in AML. ${ }^{8}$

Multiple studies have shown that the results of MRD monitoring can predict relapse in CBF-AML., ${ }^{49-18}$ Given this capacity to identify patients at risk of imminent relapse, the recently published consensus statement from the European LeukemiaNet recommends molecular MRD monitoring for CBF-AML in the peripheral blood (PB) and bone marrow (BM) at diagnosis, after two cycles of induction/consolidation chemotherapy, at the end of treatment, and every 3 months for 24 months of follow-up. ${ }^{6}$ Presumably, by monitoring patients for rising molecular transcripts, those at risk of impending relapse can be identified and treated with allogeneic bone marrow transplantation (BMT) prior to the emergence of overt disease and the need for re-induction chemotherapy. However, the relapse kinetics of CBF-AML are poorly understood, and it is not known whether serial MRD monitoring can detect molecular relapses with sufficient lead-time to intervene and prevent morphological relapse. Furthermore, it is uncertain if patients' outcomes can be improved by using $\mathrm{MRD}$ status to risk-stratify patients and guide treatment decisions such as escalation to allogeneic BMT. We therefore conducted a study to determine the relapse kinetics and clinical utility of serial MRD monitoring in CBF-AML.

\section{Methods}

\section{Study design and population}

This retrospective study included all patients $\geq 18$ years old with CBF-AML at Princess Margaret Cancer Centre in Toronto, Canada between January 2000 and 2017. Patients were excluded if they did not have MRD monitoring performed. This study was approved by the institutional Research Ethics Board.

\section{Treatment and measurable residual disease monitoring protocol}

The standard treatment protocol for eligible patients with CBFAML at our institution during the study period was induction chemotherapy with daunorubicin for 3 days and continuous infusion of cytarabine for 7 days. Patients who achieved complete remission received three cycles of consolidation with high-dose cytarabine along with daunorubicin during the first cycle of consolidation (see Online Supplementary Methods). Allogeneic BMT was not routinely recommended unless there was molecular progression during follow-up or if risk factors were present such as $\mathrm{t}(8 ; 21)$ with C-KIT mutation. ${ }^{19}$

Patients underwent MRD monitoring using RT-qPCR of RUNX1-RUNX1T1 or CBFB-MYH11 fusion transcripts in an accredited (ISO15189) laboratory at the University Health Network (see Online Supplementary Methods). Each patient's own diagnostic level was used as baseline for calculating log reduction. The detection limit for these assays was 1 in 10,000 throughout the study period.
MRD measurements were generally performed at diagnosis, after induction and the final cycle of consolidation, and then every 3 months during follow-up for 24 months. MRD assessment was routinely performed on $\mathrm{BM}$ aspirates, but $\mathrm{PB}$ was tested in patients who could not tolerate repeat BM aspirates. A molecular relapse was typically confirmed with a repeat sample 4 weeks later, with referral for allogeneic BMT or administration of chemotherapy if confirmed. Patients who developed morphological relapse were treated with re-induction chemotherapy to achieve second complete remission and referred for allogeneic BMT if a suitable donor was available. ${ }^{19}$

\section{Study protocol}

We determined the risk of relapse among patients with CBFAML and the median time from molecular relapse to morphological relapse. We empirically defined rapid relapse as $<100$ days from molecular to morphological relapse as we considered this timeframe insufficient to directly administer allogeneic BMT to prevent morphological relapse. Morphological complete remission was defined as $<5 \%$ blasts in BM with recovery of peripheral cell counts and no evidence of extramedullary disease. Morphological relapse was defined as the re-emergence of leukemic blasts in $\mathrm{PB}$, $\geq 5 \%$ blasts in $\mathrm{BM}$, or development of extramedullary disease. ${ }^{20}$ Complete molecular remission was defined as two successive MRD-negative samples $>4$ weeks apart, and molecular relapse as an increase of MRD transcript level $\geq 1$ log between two successive samples. ${ }^{6}$

\section{Statistical analysis}

We performed Student t-tests for continuous variables and a $\chi^{2}$ test/Fisher exact test for categorical variables to analyze differences between patients with remission or relapse and between those with rapid or slow relapse kinetics. A log-rank test was used to compare relapse-free survival between patients who achieved $\geq 3$ or $<3 \log$ reduction in MRD at the end of treatment. Results with a $P$ value $<0.05$ were considered statistically significant. All analyses were performed using GraphPad statistical software and version 9.4 of the SAS System for Windows (C) 2002-2012 SAS Institute, Inc., Cary, NC, USA).

\section{Results}

\section{Study population}

We identified 206 patients with CBF-AML who were evaluated at our institution during the study period. Of these 206 patients, 114 had MRD monitoring performed and were included in our analysis. We excluded 92 patients who did not have MRD monitoring available because of death during treatment $(n=19)$, administration of non-intensive or supportive care only $(n=12)$, allogeneic BMT performed in first complete remission $(n=17), M R D$ monitoring conducted at an outside institution $(n=24)$, or for unspecified reasons $(n=20)$ (Figure 1).

The median age of the 114 patients was 46.5 years (range, 18-79) and $44.7 \%$ were female. The t(8;21) was present in $58.8 \%$ and $\operatorname{inv}(16) / \mathrm{t}(16 ; 16)$ in $41.2 \%$ of patients. Over a median follow-up time of 3.7 years (range, 0.2 14.3), MRD measurements were performed a mean ( \pm standard deviation, SD) 4.9 \pm 3.2 times per patient with a median (range) sampling interval of 98 (0-378) days. A total of $564 \mathrm{MRD}$ measurements were performed, of which $77.5 \%$ within 120 days of the previous MRD sample. Most MRD measurements were performed on BM samples and $13.7 \%$ were performed on $\mathrm{PB}$. 


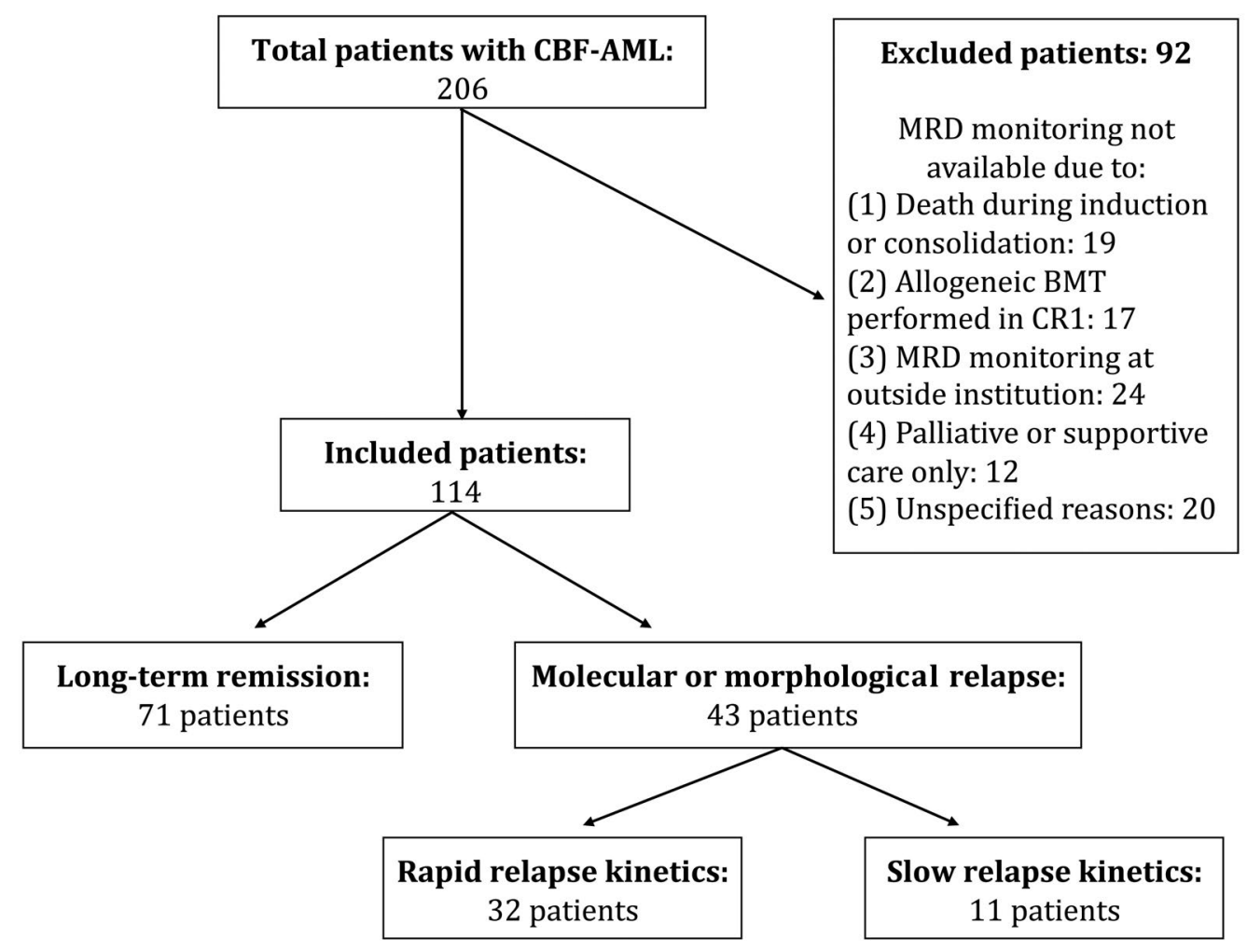

Figure 1. Flow diagram of patients with core-binding factor acute myeloid leukemia. CBF-AML: core-binding factor acute myeloid leukemia; MRD: measurable residual disease, BMT: bone marrow transplantation; CR1: first complete remission.

\section{Risk of relapse}

All patients except one $(99.1 \%)$ achieved complete remission with standard induction chemotherapy; the single patient with refractory disease achieved complete remission with re-induction chemotherapy. Long-term remission was maintained in $71(62.3 \%)$ patients but 43 $(37.7 \%)$ relapsed, of whom 34 developed morphological relapse and nine had isolated molecular relapse but did not progress to morphological relapse because of pre-emptive treatment with allogeneic BMT or death from complications of treatment or disease. Most relapses occurred early with a median time from complete remission to molecular or morphological relapse of 9.1 months (range, 1.6-38.6); only $2 / 43(4.7 \%)$ relapses occurred $>2$ years after the achievement of complete remission. The risk of relapse was significantly higher in patients who achieved $<3$ log reduction in RUNX1-RUNX1T1 or CBFB-MYH11 transcripts at the end of consolidation chemotherapy compared to patients who achieved $\geq 3$ log reduction $(61.1 \%$ vs. $33.7 \%, P=0.004$ ) (Figure 2). Patients with $<4$ log reduction in MRD transcripts at the end of treatment also had a higher risk of relapse compared to patients with $\geq 4 \log$ reduction $(51.2 \%$ vs. $29.3 \%, P=0.026)$. Furthermore, relapsed patients were significantly less likely to have achieved MRD-negative status at the end of chemotherapy $(23.1 \%$ vs. $45.2 \%, P=0.025)$ or complete molecular remission during follow-up $(7.0 \%$ vs. $74.6 \%, P<0.0001)$, compared to patients who did not relapse (Table 1). There was no significant association between risk of relapse and MRD log reduction at the end of induction or with other clinical, cytogenetic, or genetic variables. There was a trend, which bordered on statistical significance, towards more extramedullary disease in patients with relapse (Table 1).

Of the 71 patients who did not relapse, 28 (39.4\%) had persistent molecular disease during follow-up with detectable MRD at low transcript levels but with $<1$ log increase between two successive positive samples. Persistent molecular disease continued for a median of 1.5 years and up to a maximum of 3.4 years from the time of complete remission. Of these 28 patients, 13 eventually achieved MRD-negative status and 15 had persistent molecular disease to the end of follow-up MRD monitoring.

\section{Relapse kinetics}

Of the 43 patients who relapsed, the majority $(74.4 \%$, $\mathrm{n}=32)$ had rapid relapse kinetics with $<100$ days from molecular to morphological relapse. Of the 32 patients with rapid relapse kinetics, 17 (53.1\%) had simultaneous molecular and morphological relapse, meaning that they progressed from a MRD-negative status $(n=3)$ or stable levels of $\operatorname{MRD}(n=14)$ on one sample to morphological relapse on the subsequent sample. The remaining 15/32 $(46.9 \%)$ developed molecular relapse prior to morphological relapse, but progression occurred rapidly over a median of 48 days (range, 11-95) such that no pre-emptive therapy, including allogeneic BMT, could be administered. Of the patients with rapid relapse kinetics, $16(50.0 \%)$ received re-induction chemotherapy at the time of morphological relapse followed by allogeneic BMT. Eight patients $(25.0 \%)$ received additional chemotherapy but 


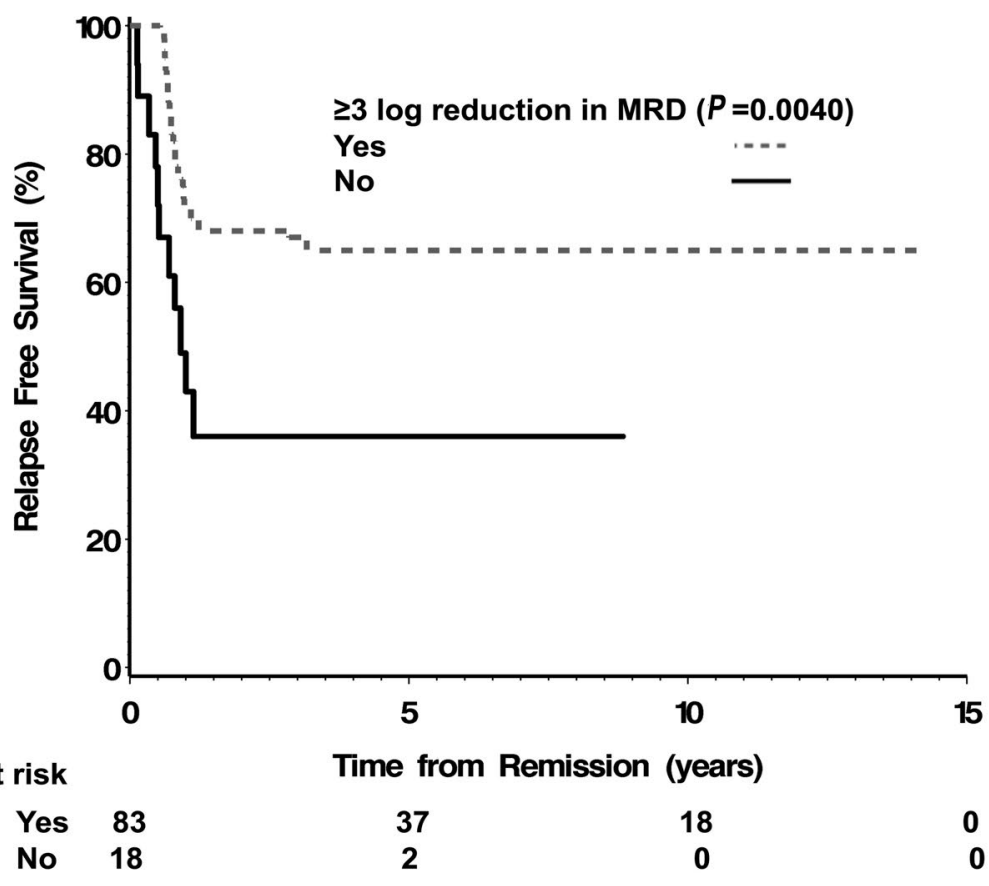

Figure 2. Kaplan-Meier analysis of relapse-free survival, according to reduction in measurable residual disease at end of chemotherapy. MRD:

Patients at risk

No 18
2 $\mathbf{0}$ did not proceed to allogeneic BMT and died from complications of chemotherapy or disease. Five (15.6\%) patients died without further chemotherapy and three $(9.4 \%)$ were lost to follow-up.

MRD monitoring enabled timely detection of molecular relapse and permitted intervention prior to morphological relapse in only 11 patients $(25.6 \%)$. Of these patients with slower relapse kinetics, eight $(72.7 \%)$ went on to receive allogeneic BMT and three $(27.3 \%)$ received additional chemotherapy but died from complications of chemotherapy or disease prior to allogeneic BMT. Pre-emptive intervention with allogeneic BMT at the time of molecular relapse prevented the development of morphological relapse in $6 / 11(54.5 \%)$ patients. The median overall survival was 33.6 months for patients with slower relapse kinetics compared to 21.7 months for patients with rapid relapse kinetics $(P=0.070)$.

There were no clinical features, molecular mutations, or cytogenetic abnormalities that significantly predicted patients with rapid versus slower relapse kinetics (Table 2). Furthermore, our inability to predict most relapses did not appear to be due to differences in MRD sampling technique, as there was no significant difference between patients with rapid and slower relapse kinetics in terms of the median time interval between MRD samples (70.5 vs. 70.0 days, $P=0.74)$, the proportion of delayed MRD measurements $(7.1 \%$ vs. $12.5 \%, P=0.35)$, or the number of $\mathrm{MRD}$ tests performed on $\mathrm{PB}(10.0 \%$ vs. $8.3 \%, P=1.0)$. Among patients who had a rise in MRD transcripts detected prior to the emergence of morphological relapse, a confirmatory sample was obtained within 4 weeks in 8/17 $(47.1 \%)$ cases in the rapid relapse group (median 31 days; range, 3-91) and in 5/8 (62.5\%) cases in the slower relapse group (median 28 days; range, 8-185), with no significant difference in time to confirmatory sample between the two groups $(P=0.68)$. The time period during which relapses occurred was also similar between the two groups, with 20/32 (62.5\%) relapses occurring after 2010 in the rapid relapse group compared to $5 / 11$ (45.5\%) in the slower relapse group $(P=0.48)$.

Finally, a secondary analysis restricted to patients who achieved an optimal molecular response (defined here as $\geq 3 \log$ reduction in $\mathrm{MRD}$ at the end of chemotherapy) yielded similar findings as our primary analysis. The majority of these patients $(67.9 \%, n=19)$ experienced rapid progression from molecular to morphological relapse (median 11 days; range, 0-95), and MRD monitoring enabled the timely detection of impending relapse in only $9 / 28(32.1 \%)$ of patients with an optimal molecular response.

\section{Discussion}

Although CBF-AML is associated with a higher rate of complete remission and a relatively favorable prognosis, up to $40 \%$ of patients develop relapse. Current international guidelines for CBF-AML recommend MRD monitoring every 3 months for 2 years after remission. This practice is intended to identify patients at risk of recurrence by detecting impending relapse and, presumably, to enable early intervention to prevent morphological relapse. However, our study demonstrates that this monitoring strategy fails to detect the majority of relapsing patients in a timely manner, as $74.4 \%$ of patients at our institution developed rapid morphological relapses such that no pre-emptive therapy, including allogeneic BMT, could be administered. In almost half these patients, molecular relapse occurred simultaneously with morphological relapse, while in the remaining cases the progression from molecular to morphological relapse occurred rapidly over a median of only 48 days. The current practice of measuring MRD every 3 months during follow-up therefore appears to provide insufficient lead-time to iden- 
tify molecular relapses and intervene prior to morphological relapse. As a consequence, 114 patients in our study underwent serial MRD monitoring with BM sampling every 3 months for 24 months, but only 11 patients $(9.6 \%)$ experienced a direct change in clinical management from this practice by receiving pre-emptive treatment prior to morphological relapse. This suggests that MRD monitoring may be of rare clinical utility in the follow-up of patients with CBF-AML.

The results of our study are significant given the lack of large longitudinal studies examining the clinical utility of serial MRD monitoring in CBF-AML. Our results are consistent with the long-term follow-up reported from the UK MRC AML-15 trial, in which serial MRD monitoring failed to predict impending relapse in $42 / 71(59.2 \%)$ patients with morphological relapse. ${ }^{11}$ However, the authors of that study attributed the inability to predict impending relapse to infrequent $\mathrm{MRD}$ measurements, as sampling intervals were $>3$ months in many patients. This contrasts with the generally good MRD monitoring adherence in our population of patients, among whom the median MRD sampling interval was 98 days. However, a limitation of our study is its real-world retrospective design, and we cannot exclude that delays in $M R D$ sampling or variations in specimen quality may have limited our ability to predict impending relapse in some patients. Furthermore, the majority of relapses in our study occurred early at a median of 9 months after remission, which may have further limited the ability of serial MRD monitoring to predict relapse. Other studies have found serial MRD monitoring to be more strongly predictive of impending relapse. In a follow-up analysis of 94 patients enrolled in the French CBF-2006 trial who underwent $\mathrm{MRD}$ monitoring of $\mathrm{PB}$ every 3 months after remission, morphological relapse was predicted by persistent MRD positivity or molecular relapse in 21/29

Table 1. Analysis of patients with long-term remission versus relapse.

\begin{tabular}{|c|c|c|c|c|}
\hline Variable & & Remission ( $n=71)$ & Relapse ( $n=43)$ & $P$ value \\
\hline \multicolumn{5}{|l|}{ Clinical variables } \\
\hline Sex, n (\%) & Female & $31(43.7 \%)$ & $20(46.5 \%)$ & 0.77 \\
\hline Age at diagnosis, years & Median (range) & $47(18-72)$ & $45(19-79)$ & 0.83 \\
\hline WBC count at diagnosis, $\times 10^{9} / \mathrm{L}$ & Median (Range) & $8.0(0.90-360)$ & $9.9(1.3-341)$ & 0.24 \\
\hline BM blasts at diagnosis; $\%$ & Median (Range) & $53.5(12-95)$ & $67(15-100)$ & 0.35 \\
\hline \multirow[t]{2}{*}{ AML diagnosis, n (\%) } & De novo & $65(91.5 \%)$ & $38(88.4 \%)$ & 0.50 \\
\hline & Secondary & $5(7.1 \%)$ & $5(11.6 \%)$ & \\
\hline CNS disease, $\mathrm{n}(\%)$ & Yes & $1(1.4 \%)$ & $3(7.0 \%)$ & 0.15 \\
\hline Extramedullary disease, n (\%) & Yes & $4(5.6 \%)$ & $8(18.6 \%)$ & 0.055 \\
\hline \multicolumn{5}{|l|}{ Cytogenetic abnormalities, n (\%) } \\
\hline $\operatorname{inv}(16)$ & Yes & $32(45.1 \%)$ & $15(34.9 \%)$ & 0.28 \\
\hline $\mathrm{t}(8 ; 21)$ & Yes & $39(54.9 \%)$ & $28(65.1 \%)$ & 0.28 \\
\hline $\operatorname{del}(X)$ & Yes & $4(5.6 \%)$ & $6(14 \%)$ & 0.17 \\
\hline $\operatorname{del}(\mathrm{Y})$ & Yes & $14(19.7 \%)$ & $8(18.6 \%)$ & 0.88 \\
\hline $\operatorname{del}(9)$ & Yes & $5(7.0 \%)$ & $2(4.7 \%)$ & 0.71 \\
\hline $\operatorname{tri}(8)$ & Yes & $8(11.3 \%)$ & $2(4.7 \%)$ & 0.32 \\
\hline $\operatorname{tri}(9)$ & Yes & $3(4.2 \%)$ & $0(0 \%)$ & 0.29 \\
\hline $\operatorname{tri}(21)$ & Yes & $3(4.2 \%)$ & $1(2.3 \%)$ & 1.00 \\
\hline $\operatorname{tri}(22)$ & Yes & $6(8.5 \%)$ & $0(0 \%)$ & 0.082 \\
\hline$\geq 3$ cytogenetic abnormalities & Yes & $14(19.7 \%)$ & $5(11.6 \%)$ & 0.26 \\
\hline \multicolumn{5}{|l|}{ Genetic mutations, n (\%) } \\
\hline C-KIT mutation & Yes & $7 / 39(17.9 \%)$ & $7 / 30(23.3 \%)$ & 0.58 \\
\hline NPM1 mutation & Yes & $0 / 2(0 \%)$ & $0 / 4(0 \%)$ & $\mathrm{NA}$ \\
\hline FLT3-ITD mutation & Yes & $1 / 2(50 \%)$ & $0 / 4(0 \%)$ & 0.33 \\
\hline FLT3-TKD mutation & Yes & $0 / 2(0 \%)$ & $1 / 4(25 \%)$ & 1.00 \\
\hline \multicolumn{5}{|l|}{ Outcomes } \\
\hline CR to induction, $\mathrm{n}(\%)$ & Yes & $71(100 \%)$ & $42(97.7 \%)$ & 0.38 \\
\hline CR to consolidation, $\mathrm{n}(\%)$ & Yes & $71(100 \%)$ & $39(90.7 \%)$ & 0.02 \\
\hline Morphological relapse, $n(\%)$ & Yes & $0(0)$ & $34(79.1 \%)$ & $<0.0001$ \\
\hline Duration of CR, months & Median (range) & $87.1(10.2-173.4)$ & $9.1(1.6-38.6)$ & $<0.0001$ \\
\hline Death & Yes & $3(4.2 \%)$ & $30(69.8 \%)$ & $<0.0001$ \\
\hline \multicolumn{5}{|l|}{ MRD status } \\
\hline End of induction MRD $\geq 3$ log reduction, $n(\%)$ & Yes & $25 / 42(59.5 \%)$ & 15/33 (45.5\%) & 0.23 \\
\hline End of consolidation MRD $\geq 3$ log reduction, $n(\%)$ & Yes & 55/62 (88.7\%) & 28/39 (71.8\%) & 0.031 \\
\hline End of consolidation MRD $\geq 4 \log$ reduction, $n(\%)$ & Yes & 41/62 (66.1\%) & 17/39 (43.6\%) & 0.038 \\
\hline End of consolidation MRD negative, $\mathrm{n}(\%)$ & Yes & $28 / 62(45.2 \%)$ & $9 / 39(23.1 \%)$ & 0.025 \\
\hline Complete molecular remission during follow-up, n (\%) & Yes & $53 / 71(74.6 \%)$ & $3 / 43(7.0 \%)$ & $<0.0001$ \\
\hline Number of MRD tests per patient, $\mathrm{n}(\%)$ & Mean (SD) & $6.3(3.1)$ & $2.7(2.2)$ & $<0.0001$ \\
\hline Number of MRD tests performed on PB, $n(\%)$ & Sum & $66 / 446(14.8 \%)$ & $11 / 118(9.3 \%)$ & 0.13 \\
\hline Time between MRD tests, days & Median (range) & $109(26.3-378)$ & $70(0-164)$ & $<0.0001$ \\
\hline Number of MRD tests performed $>120$ days apart, $\mathrm{n}(\%)$ & Sum & $116 / 446(26.0 \%)$ & $11 / 118(9.3 \%)$ & $<0.0001$ \\
\hline
\end{tabular}

WBC: white blood cell count; BM: bone marrow; AML: acute myeloid leukemia; CNS: central nervous system; CR: complete remission; NA: not available; ITD: internal tandem duplication; TKD: tyrosine kinase domain; MRD: measurable residual disease; PB: peripheral blood; SD: standard deviation. 
$(72.4 \%)$ cases. ${ }^{21}$ However, it is unclear if the timelier detection of impending relapse in that study translated into early intervention and improved clinical outcomes, as the cumulative incidence of morphological relapse remained high at $74.5 \%$ among the 23 patients with molecular relapse.

This issue is further complicated by incomplete understanding of the relapse kinetics and optimal sampling interval of MRD measurements in CBF-AML. The current recommendation of $\mathrm{MRD}$ monitoring every 3 months is derived from the UK MRC AML-15 trial which reported a median time from molecular relapse in the BM to mor- phological relapse of 4.9 months in cases with $t(8 ; 21)$ and 3 months in those with inv(16). ${ }^{11}$ Similarly, the CBF-2006 trial demonstrated a median time from molecular to morphological relapse of 3.9 months. ${ }^{21}$ However, other studies have described slower relapse kinetics with a median time from molecular to morphological relapse of up to 6 months or even 1 year in patients with $\operatorname{inv}(16)^{22,23}$ Alternatively, other analyses are consistent with the rapid relapse kinetics observed in our study, with reported median times from molecular to morphological relapse of 35 to 60 days in small studies. ${ }^{24,25}$ The discordant relapse kinetics across studies may be attributable to differences

Table 2. Analysis of patients with rapid versus slow relapse kinetics.

\begin{tabular}{|c|c|c|c|c|}
\hline Variable & Level & $\begin{array}{c}\text { Rapid relapse } \\
(n=32)\end{array}$ & $\begin{array}{c}\text { Slow relapse } \\
(\mathrm{n}=\mathbf{1 1})\end{array}$ & $P$ value \\
\hline $\begin{array}{l}\text { Clinical variables } \\
\text { Sex, } \mathrm{n}(\%) \\
\text { Age at diagnosis, years } \\
\text { WBC count at diagnosis, x10\%/ } \\
\text { BM blasts at diagnosis, } \% \\
\text { Diagnosis, } \mathrm{n}(\%) \\
\text { CNS disease, } \mathrm{n}(\%) \\
\text { Extramedullary disease, } \mathrm{n}(\%)\end{array}$ & $\begin{array}{c}\text { Female } \\
\text { Median (Range) } \\
\text { Median (Range) } \\
\text { Median (Range) } \\
\text { De novo AML } \\
\text { Secondary AML } \\
\text { Yes } \\
\text { Yes }\end{array}$ & $\begin{array}{l}15(46.9 \%) \\
45(19-79) \\
9.8(1.3-198) \\
67(15-93) \\
27(84.4 \%) \\
5(15.6 \%) \\
1(3.1 \%) \\
5(15.6 \%)\end{array}$ & $\begin{array}{l}5(45.5 \%) \\
41(24-59) \\
10.1(3.7-341) \\
68(20-100) \\
11(100 \%) \\
0(0 \%) \\
2(18.2 \%) \\
3(27.3 \%)\end{array}$ & $\begin{array}{l}0.94 \\
0.64 \\
0.27 \\
0.35 \\
0.31 \\
\\
0.16 \\
0.40\end{array}$ \\
\hline $\begin{array}{l}\text { Cytogenetic abnormalities, n (\%) } \\
\text { inv }(16) \\
\text { t( }(8 ; 21) \\
\operatorname{del}(X) \\
\operatorname{del}(Y) \\
\operatorname{del}(9) \\
\operatorname{tri}(8) \\
\operatorname{tri}(9) \\
\operatorname{tri}(21) \\
\text { tri }(22) \\
\geq 3 \text { cytogenetic abnormalities }\end{array}$ & $\begin{array}{l}\text { Present } \\
\text { Present } \\
\text { Present } \\
\text { Present } \\
\text { Present } \\
\text { Present } \\
\text { Present } \\
\text { Present } \\
\text { Present } \\
\text { Present }\end{array}$ & $\begin{array}{c}9(28.1 \%) \\
23(71.9 \%) \\
4(12.5 \%) \\
7(21.9 \%) \\
1(3.1 \%) \\
2(6.3 \%) \\
0(0 \%) \\
1(3.1 \%) \\
0(0 \%) \\
4(12.5 \%)\end{array}$ & $\begin{array}{l}6(54.6 \%) \\
5(45.5 \%) \\
2(18.2 \%) \\
1(9.1 \%) \\
1(9.1 \%) \\
0(0 \%) \\
0(0 \%) \\
0(0 \%) \\
0(0 \%) \\
1(9.1 \%)\end{array}$ & $\begin{array}{l}0.15 \\
0.15 \\
0.64 \\
0.66 \\
0.45 \\
1.00 \\
\text { NA } \\
1.00 \\
\text { NA } \\
1.00\end{array}$ \\
\hline $\begin{array}{l}\text { Genetic mutations, n (\%) } \\
\text { CKIT mutation } \\
\text { NPM1 mutation } \\
\text { FLT3-ITD mutation } \\
\text { FLT3-TKD mutation }\end{array}$ & $\begin{array}{l}\text { Present } \\
\text { Present } \\
\text { Present } \\
\text { Present }\end{array}$ & $\begin{array}{l}6 / 24(25 \%) \\
0 / 3(0 \%) \\
0 / 3(0 \%) \\
0 / 3(0 \%)\end{array}$ & $\begin{array}{l}1 / 6(16.7 \%) \\
0 / 1(0 \%) \\
0 / 1(0 \%) \\
1 / 1(100 \%)\end{array}$ & $\begin{array}{l}1.00 \\
\text { NA } \\
\text { NA } \\
0.25\end{array}$ \\
\hline $\begin{array}{l}\text { Treatment } \\
\text { Induction chemotherapy, n (\%) } \\
\text { Consolidation chemotherapy, median (range) } \\
\text { Allogeneic BMT }\end{array}$ & $\begin{array}{c}7+3 \\
\text { Cycles } \\
\text { Performed }\end{array}$ & $\begin{array}{c}32(100 \%) \\
3(1-4) \\
17(53.1 \%)\end{array}$ & $\begin{array}{l}11(100 \%) \\
3(2-4) \\
8(72.7 \%)\end{array}$ & $\begin{array}{l}\text { NA } \\
0.41 \\
0.31\end{array}$ \\
\hline $\begin{array}{l}\text { Outcomes } \\
\text { CR to induction, n (\%) } \\
\text { CR to consolidation, n (\%) } \\
\text { Morphologic relapse, n (\%) } \\
\text { Molecular relapse before morphological } \\
\text { relapse, n (\%) } \\
\text { Duration of CR, months } \\
\text { Death, n (\%) }\end{array}$ & $\begin{array}{c}\text { Yes } \\
\text { Yes } \\
\text { Yes } \\
\text { Yes } \\
\text { Median (range) } \\
\text { Yes }\end{array}$ & $\begin{array}{c}31(96.9 \%) \\
28(87.5 \%) \\
32(100 \%) \\
\\
15(46.9 \%) \\
9.0(1.6-34.7) \\
24(75.0 \%)\end{array}$ & $\begin{array}{c}11(100 \%) \\
11(100 \%) \\
2(18.2 \%) \\
\\
11(100 \%) \\
9.8(7.6-38.6) \\
6(54.5 \%)\end{array}$ & $\begin{array}{c}1.00 \\
0.56 \\
<0.0001 \\
\\
0.001 \\
0.22 \\
0.26\end{array}$ \\
\hline $\begin{array}{l}\text { MRD status } \\
\text { End of induction } M R D \geq 3 \text { log reduction, } n \text { (\%) } \\
\text { End of consolidation } M R D \geq 3 \text { log reduction, } n(\%) \\
\text { End of consolidation MRD negative, } n(\%) \\
\text { Number of MRD tests/patient } \\
\text { Number of MRD tests performed on } \mathrm{PB}, \mathrm{n}(\%) \\
\text { Sampling interval between MRD testing, days } \\
\text { Number of MRD tests performed } \\
>120 \text { days apart, } \mathrm{n}(\%)\end{array}$ & $\begin{array}{c}\text { Yes } \\
\text { Yes } \\
\text { Yes } \\
\text { Mean (SD) } \\
\text { Sum } \\
\text { Median (range) }\end{array}$ & $\begin{array}{c}11 / 25(44.0 \%) \\
19 / 29(65.5 \%) \\
6 / 29(20.7 \%) \\
2.2(1.7) \\
7 / 70(10.0 \%) \\
70.5(0-126)\end{array}$ & $\begin{array}{c}4 / 8(50.0 \%) \\
9 / 10(90.0 \%) \\
3 / 10(30.0 \%) \\
4.3(2.7) \\
4 / 48(8.3 \%) \\
70(46-164)\end{array}$ & $\begin{array}{l}1.00 \\
0.23 \\
0.67 \\
0.004 \\
1.00 \\
0.74 \\
0.35\end{array}$ \\
\hline
\end{tabular}

WBC: white blood cell count; BM: bone marrow; AML: acute myeloid leukemia; CNS: central nervous system; CR: complete remission; NA: not available; ITD: internal tandem duplication; TKD: tyrosine kinase domain; MRD: measurable residual disease; PB: peripheral blood; SD: standard deviation. 
in sampling source (i.e., $\mathrm{PB}$ or $\mathrm{BM}$ ), variable sensitivity of individual RT-qPCR assays, or differing MRD sampling intervals. ${ }^{26}$ Individual patient-level factors may also contribute to differences in relapse kinetics, although our study did not identify any clinical variables significantly predictive of rapid relapse kinetics. Additional research is therefore warranted to better characterize the relapse kinetics of CBF-AML and to determine whether more frequent MRD sampling (e.g., PB every 4-8 weeks) might be more predictive of impending relapse.

An additional area of uncertainty and interest is whether serial MRD monitoring can be used to inform treatment decisions. Currently, the European LeukemiaNet does not formally recommend changing therapy based on MRD status in CBF-AML due to lack of supportive data. ${ }^{6}$ However, the AML05 trial demonstrated that utilizing MRD status to identify high-risk patients with $t(8 ; 21)$ (i.e., those with $<3$ $\log$ reduction in MRD after the second cycle of consolidation or loss of molecular response within 6 months) as candidates for escalation of therapy to allogeneic BMT in first complete remission may lead to improved clinical outcomes. ${ }^{27}$ The GIMEMA AML1310 study also provides support for a risk-adapted, MRD-directed treatment strategy, as patients with intermediate-risk AML who were MRDpositive at the end of the first cycle of consolidation chemotherapy were referred for allogeneic BMT and achieved similar disease-free and overall survival rates as those with favorable-risk AML ${ }^{28}$ In the present study, preemptive intervention with allogeneic BMT at the time of molecular relapse prevented the development of morphological relapse in 6/11 patients with slower relapse kinetics. However, this finding requires confirmation in other populations of patients given the relatively small size and retrospective design of our study. The role for pre-emptive chemotherapy or allogeneic BMT in CBF-AML is also uncertain because most patients with morphological relapse respond well to salvage treatments. ${ }^{26}$ Future studies should examine the clinical outcomes of different treatment strategies (e.g., observation or pre-emptive chemotherapy, immunotherapy, or BMT) for patients with CBF-AML in molecular relapse.

Although our study raises questions about the utility of serial MRD monitoring during follow-up after remission, we did identify the prognostic importance of measuring $M R D$ at the end of treatment. In our study population, achievement of $\geq 3$ log reduction in RUNX1-RUNX1T1 or CBFB-MYH11 transcripts at this time-point was associated with a significantly lower risk of relapse. This is consistent with the findings of other trials, including the UK MRC AML-15, AML05, and CBF-2006 trials, in which achievement of deep reductions in MRD transcript levels after induction or consolidation chemotherapy was associated with improved relapse-free survival.11,12,27,29 Our study did not analyze the prognostic significance of measuring MRD after the second cycle of chemotherapy as recommended by the European LeukemiaNet, as the local institutional policy during this study period recommended measuring $\mathrm{MRD}$ after induction and the final cycle of consolidation chemotherapy. Other studies have identified additional potential prognostic factors that may predict relapse and poor outcomes in CBF-AML, including older age, elevated white blood cell count at diagnosis, extramedullary disease, secondary AML, additional cytogenetic changes (e.g., loss of chromosome X or Y, trisomy 22 , deletion of 9q), and genetic mutations (e.g., KIT, FLT3, RAS, ASXL). ${ }^{3}$

In conclusion, measuring $\mathrm{MRD}$ at the end of treatment is predictive of relapse risk in CBF-AML. However, the current guideline recommendation of MRD monitoring every 3 months during follow-up failed to detect the majority of molecular relapses with sufficient lead-time to intervene and prevent morphological relapse in our study population. Further research is warranted to characterize the relapse kinetics of CBF-AML and to identify the patients at highest risk of relapse and the optimal strategies to monitor these patients over time.

\section{Disclosures}

$A D S$ has received honorarium from Novartis, Jazz, and Otsuka Pharmaceuticals and research support from Medivir $A B$ and Takeda. A.D.S. is named as an inventor on a patent application related to the use of DNT cells in AML. A.D.S. owns stock in AbbVie Pharmaceuticals. VG received honorarium, research funding through institution, and served on advisory board of Novartis and Incyte. CM has received honorarium from Novartis.

\section{Contributions}

$R P$ performed research, performed data analysis, and wrote the manuscript. EA performed data analysis. JO, SC,VG, DM, $C M, T M, A S, K Y, H S, M M, C W, T S$, and SK contributed to performing research and writing the manuscript. AS supervised the study and contributed to writing the manuscript.

\section{Acknowledgments}

We thank Jill Flewelling (Princess Margaret Cancer Center) for administrative assistance. This work was supported by the Canadian Institutes of Health Research, the Princess Margaret Cancer Centre, The Princess Margaret Cancer Foundation, and the Ontario Ministry of Health. ADS holds the Ronald N. Buick Chair in Oncology Research.

\section{References}

1. Arber DA, Orazi A, Hasserjian R, et al. The 2016 revision to the World Health Organization classification of myeloid neoplasms and acute leukemia. Blood. 2016;127(20):2391-2405.

2. Grimwade D, Hills RK, Moorman AV, et al. Refinement of cytogenetic classification in acute myeloid leukemia: determination of prognostic significance of rare recurring chromosomal abnormalities among 5876 younger adult patients treated in the
United Kingdom Medical Research Council trials. Blood. 2010;116(3):354-365.

3. Duployez N, Willekens C, Marceau-Renaut A, et al. Prognosis and monitoring of corebinding factor acute myeloid leukemia: current and emerging factors. Expert Rev Hematol. 2015;8(1):43-56.

4. Ustun $C$ and Marcucci G. Emerging diagnostic and therapeutic approaches in core binding factor acute myeloid leukaemia. Curr Opin Hematol. 2015;22(2):85-91.

5. Schlenk RF, Benner A, Krauter J, et al. Individual patient data-based meta-analysis of patients aged 16 to 60 years with core binding factor acute myeloid leukemia: survey of the German Acute Myeloid Leukemia Intergroup. J Clin Oncol. 2004;22(18):3741-3750

6. Schuurhuis GJ, Heuser M, Freeman S, et al Minimal/measurable residual disease in AML: a consensus document from the European LeukemiaNet MRD Working Party. Blood. 2018;131(12): 1275-1291.

7. Paietta E. Minimal residual disease in acute myeloid leukemia: coming of age. Hematology Am Soc Hematol Educ Program. 2012;2012:35-42.

8. Ofran Y, Rowe JM. Introducing minimal 
residual disease in acute myeloid leukemia. Curr Opin Hematol. 2015;22(2): 139-145.

9. Deotare U, Shaheen M, Brandwein JM, et al. Predictive value of molecular remissions postconsolidation chemotherapy in patients with core binding factor acute myeloid leukemia (CBF-AML) - a single center analysis. Hematol Oncol. 2016;35(4): 810-813.

10. Hoyos M, Nomdedeu JF, Esteve J, et al. Core binding factor acute myeloid leukemia: the impact of age, leukocyte count, molecular findings, and minimal residual disease. Eur J Haematol. 2013;91(3):209-218.

11. Yin JA, O'Brien MA, Hills RK, et al. Minimal residual disease monitoring by quantitative RT-PCR in core binding factor AML allows risk stratification and predicts relapse: results of the United Kingdom MRC AML-15 trial. Blood. 2012;120(14): 2826-2835

12. Jourdan E, Boissel N, Chevret S, et al. Prospective evaluation of gene mutations and minimal residual disease in patients with core binding factor acute myeloid leukemia. Blood. 2013;121(12):2213-2223.

13. Zhang L, Li O, Li W, et al. Monitoring of minimal residual disease in acute myeloid leukemia with $\mathrm{t}(8 ; 21)(\mathrm{q} 22 ; \mathrm{q} 22)$. Int $\mathrm{J}$ Hematol. 2013;97(6):786-792.

14. Guieze R, Renneville A, Cayuela JM, et al. Prognostic value of minimal residual disease by real-time quantitative $\mathrm{PCR}$ in acute myeloid leukemia with CBFB-MYH11 rearrangement: the French experience. Leukemia. 2010;24(7):1386-1388.

15. Corbacioglu A, Scholl C, Schlenk RF, et al. Prognostic impact of minimal residual disease in CBFB-MYH11-positive acute myeloid leukemia. J Clin Oncol. 2010;28(23):3724-3729.

16. Markova J, Markova J, Trnkova Z, et al. Monitoring of minimal residual disease in patients with core binding factor acute myeloid leukemia and the impact of C-KIT, FLT3, and JAK2 mutations on clinical outcome. Leuk Lymphoma. 2009;50(9):14481460.

17. Weisser M, Haferlach C, Hiddeman W, et al. The quality of molecular response to chemotherapy is predictive for the outcome of AML1-ETO-positive AML and is independent of pretreatment risk factors. Leukemia. 2007;21(6):1177-1182.

18. Krauter J, Gorlich K, Ottmann O, et al Prognostic value of minimal residual disease quantification by real-time reverse transcriptase polymerase chain reaction in patients with core binding factor leukemias. J Clin Oncol. 2003;21(23):4413-4422.

19. Leukemia Site Group. Acute myeloid leukemia. Princess Margaret Cancer Centre Clinical Practice Guidelines. 2015; https://www.uhn.ca/PrincessMargaret/He alth_Professionals/Programs_Departments /Leukemia/Documents/CPG_Leukemia_A cuteMyeloidLeukemia.pdf [Accessed September 8, 2020]

20. Cheson BD, Bennet JM, Kopecky KJ, et al. Revised Recommendations of the International Working Group for Diagnosis, Standardization of Response Criteria, Treatment Outcomes, and Reporting Standards for Therapeutic Trials in Acute Myeloid Leukemia. J Clin Oncol. 2003;21 (24):4642-4649

21. Willekens $C$, Blanchet $O$, Renneville A, et al. Prospective long-term minimal residual disease monitoring using RQ-PCR in RUNX1RUNX1T1-positive acute myeloid leukemia: results of the French CBF-2006 trial. Haematologica. 2016;101(3):328-335

2. Ommen HB, Schnittger S, Jovanovic JV, et al. Strikingly different molecular relapse kinetics in NPM1c, PML-RARA, RUNX1RUNX1T1, and CBFB-MYH11 acute myeloid leukemias. Blood. 2010;115(2):198-
205.

23. Stentoft J, Hokland P, Ostergaard M, et al. Minimal residual core binding factor AMLs by real time quantitative PCR--initial response to chemotherapy predicts event free survival and close monitoring of peripheral blood unravels the kinetics of relapse. Leuk Res. 2006;30(4):389-395.

24. Doubek M, Palasek I, Pospisil Z, et al Detection and treatment of molecular relapse in acute myeloid leukemia with RUNX1 (AML1), CBFB, or MLL gene translocations: frequent quantitative monitoring of molecular markers in different compartments and correlation with WT1 gene expression. Exp Hematol. 2009;37(6): 659-672.

25. Lane S, Saal R, Mollee P, et al. A >or=1 log rise in RQ-PCR transcript levels defines molecular relapse in core binding factor acute myeloid leukemia and predicts subsequent morphologic relapse. Leuk Lymphoma. 2008;49(3):517-523.

26. Kayser S, Walter RB, Stock W, et al. Minimal residual disease in acute myeloid leukemiacurrent status and future perspectives. Curr Hematol Malig Rep. 2015;10(2):132-44.

27. Zhu HH, Zhang XH, Oin YZ, et al. MRD directed risk stratification treatment may improve outcomes of $t(8 ; 21)$ AML in the first complete remission: results from the AML05 multicenter trial. Blood. 2013;121 (20):4056-4062.

28. Venditti A, Piciocchi A, Candoni A, et al. GIMEMA AML1310 trial of risk-adapted, MRD-directed therapy for young adults with newly diagnosed acute myeloid leukemia. Blood. 2019;132(12):935-945.

29. Agrawal M, Corbacioglu A, Paschka P, et al. Minimal residual disease monitoring in acute myeloid leukemia (AML) with translocation $\mathrm{t}(8 ; 21)(\mathrm{q} 22 ; \mathrm{q} 22)$ : results of the AML Study Group (AMLSG) [Abstract]. Blood. 2016;128(22):1207. 